\title{
Rehabilitation and Intensive Care Unit
}

\section{Deokkyu Kim}

Critically ill patients frequently experience one or more life-threatening organ failures such as cardiovascular, pulmonary, renal, hepatic failure, etc. Intensive care that focuses on the recovery of these important organs can lead to disregard for relatively less important problems. One of the problems that has been overlooked is the neuromuscular disorder in critically ill patients, and the prevalence of neuromuscular abnormalities and weakness is likely larger than generally considered [1]. Intensive care unit (ICU)-acquired neuromuscular abnormality or ICU-acquired weakness (ICUAW) is related to several factors including long duration of immobility, poor nutritional support, mechanical ventilation, and sepsis. Also, the use of sedative and neuromuscular blocking agent to prevent for fighting against mechanical ventilation cause the limitations of unexpected physical function, resulting in muscle weakness. In the 1-year study for 109 survivors after acute respiratory distress syndrome, Herridge et al. [2] reported that most patients have extrapulmonary symptoms, which were caused by muscle weakness and fatigue. Only half of the patients in this study were employed at 1 year after the discharge from ICU, and the reasons for unemployment included persistent fatigue, weakness, poor ability to exercise, and functional limitations such as foot drop, immobility of joints, and decreases with the distance walked in the other half the patients. Clinical recovery from acute and critical illness requires several days to weeks, but physical recovery from weight loss, muscle weakness, and physical limitation may require months to years $[2,3]$.

It is also an important point that ICUAW develops at an early phase in patients with mechanical ventilation. Leijten et al. [1] reported that more than half of patients who had mechanical ventilation for more than 7 days developed electrophysiologic abnormalities. Because of early occurrence of weakness and difficulty of recovery, the key of management for ICUAW is considered for prevention and treatment at the same time. The risk factor of ICUAW should be evaluated regularly and minimized as much as possible. The risk factors for ICUAW are as follows: unable to get

\section{Deokkyu Kim}

Department of Anesthesiology and Pain Medicine, Chonbuk National University Hospital, 20 Geonji-ro, Deokjin-gu, Jeonju 54907 , Korea

Tel: $+82-63-250-1241$

Fax: +82-63-250-1240

E-mail: aneduke@jbnu.ac.kr

ORCID

Deokkyu Kim

https://orcid.org/0000-0001-7613-3529

*No potential conflict of interest relevant to this article was reported. 
out of bed independently, anticipated long duration of critical care stay, obvious significant physical or neurological injury, lack of cognitive functioning to continue exercise independently, unable to self-ventilate on $35 \%$ of oxygen or less, presence of premorbid respiratory or mobility problems, and unable to mobilize independently over short distances [4]. Patients at risk of ICUAW should be immediately identified for what rehabilitation intervention is needed, and the application of intervention start as early as clinically possible.

Application of an early rehabilitation intervention requires several considerations about potential barriers, feasibility, safety, and necessary resources [5]. Insufficient human resource, deep sedation and a lack of knowledge regarding benefits to critically ill patients are among the most important potential barriers to successful application of early exercise intervention. Because the systemic cooperation among multidisciplinary medical staff and collaborators needed to achieve early rehabilitation, the development of ICU-specific protocols and order sets is required in the busy ICU environment. Traditionally, an exercise intervention aimed at standing ambulation was performed. For ambulation, especially with mechanically ventilated patients, many staff and equipment are required. Such equipment includes a portable cardiac monitor and pulse oximeter to allow continuous vital sign monitoring during ambulation, and a wheeled pole with infusion pumps for intravenous medications that cannot be temporarily stopped during mobilization [6]. A walker, in addition to hands-on assistance from a physical staff, provides balance and support during ambulation. A wheelchair is generally pushed behind an ambulating ICU patient to permit the patient to immediately sit and rest when necessary, and to transport patients to their room if they become physically incapable of walking due to weakness, fatigue, or medical complications. In recent years, muscular electrical stimulation (MES) and bedside cyclic ergometer are applied because these interventions require less human resource and equipment. The effectiveness of each method has already been demonstrated in several studies; it is still unclear whether the simultaneous appli- cation of the two interventions is more effective than the one performed alone. In the first study for the effect of bedside cycling alone and cycling with MES in Korea, Woo et al. [7] showed that exercise rehabilitation intervention composed of bedside cycling leads to increase of lower limb muscle mass measured by ultrasound. And they discussed that Korean ICUs don't have enough human resources for rehabilitation such as nursing, respiratory, physical therapist, occupational therapist. Compared with ambulation, in-bed cycling is very easy to apply to the critically ill patients, and the expected adverse events are few and rare. They expected synergies when additional MES was applied; there are no additional benefits according to MES application. The authors mentioned that the number of times MES is applied or the degree of electrical stimulation may not be sufficient to change the muscle mass. Also, the muscle mass was measured directly after the exercise and MES, therefore, temporary increase of perfusion and muscle mass was reflected in ultrasound measurement. Future studies are needed to evaluate effectiveness of bedside cycle with muscle electrical stimulation.

Potential benefits for patients with early exercise intervention in the ICU include improved muscle strength, physical function and quality of life. Such exercise rehabilitation intervention, bedside cycling and muscle electrical stimulation, may also decrease duration of the length of stay in ICU and hospital stay after discharge from ICU, reduce the rate of hospital readmission, and decrease the use of primary care resources. Furthermore, the systemic rehabilitation strategies could help patients return to their daily life sooner.

\section{REFERENCES}

1. Leijten FS, Harinck-de Weerd JE, Poortvliet DC, de Weerd AW. The role of polyneuropathy in motor convalescence after prolonged mechanical ventilation. JAMA 1995;274:1221-5.

2. Herridge MS, Cheung AM, Tansey CM, Matte-Martyn 
A, Diaz-Granados N, Al-Saidi F, et al. One-year outcomes in survivors of the acute respiratory distress syndrome. N Engl J Med 2003;348:683-93.

3. Cheung AM, Tansey CM, Tomlinson G, Diaz-Granados N, Matté A, Barr A, et al. Two-year outcomes, health care use, and costs of survivors of acute respiratory distress syndrome. Am J Respir Crit Care Med 2006;174:538-44.

4. National Institute for Health and Care Excellence. Rehabilitation after critical illness in adults. London: NICE; 2009.

5. Parker A, Sricharoenchai T, Needham DM. Early reha- bilitation in the intensive care unit: preventing physical and mental health impairments. Curr Phys Med Rehabil Rep 2013;1:307-14.

6. Needham DM, Truong AD, Fan E. Technology to enhance physical rehabilitation of critically ill patients. Crit Care Med 2009;37(10 Suppl):S436-41.

7. Woo K, Kim J, Kim HB, Choi H, Kim K, Lee D, et al. The effect of electrical muscle stimulation and in-bed cycling on muscle strength and mass of mechanically ventilated patients: a pilot study. Acute Crit Care 2018;33:16-22. 